\title{
Asymmetries in the Littlest Higgs Model
}

\author{
F. M. L. de Almeida Jr., Y. A. Coutinho, J. A. Martins Simões, A. J. Ramalho, S. Wulck, \\ Instituto de Física, \\ Universidade Federal do Rio de Janeiro, \\ 21941-972, Rio de Janeiro, RJ, Brazil
}

and M. A. B. do Vale

Departamento de Ciências Naturais, Universidade Federal de São João Del Rei, 36301-160, São João del Rei, MG, Brazil

Received on 27 March, 2006

\begin{abstract}
Little Higgs models were recently proposed as an alternative for models of electroweak symmetry breaking. They can be regarded as one of the important candidates of the new physics beyond the Standard Model. We consider here the phenomenology of the minimal model of this type, the "Littlest Higgs Model" (LHM). It predicts the existence of the new gauge bosons $Z_{H}$ and $A_{H}$. We calculate the contributions of these new particles to the forward-backward and left-right asymmetries in the processes $e^{+}+e^{-} \longrightarrow f+\bar{f}$. We study the possibility of detecting the lightest new gauge boson, $A_{H}$, in the future e+e- colliders with c.m. energies of $500 \mathrm{GeV}$ and 1 $\mathrm{TeV}$ and compare the LHM predictions with other models.
\end{abstract}

Keywords: Littlest Higgs; New Gauge Bosons; Asymmetries

\section{INTRODUCTION}

The Higgs divergent radiative corrections indicate that the Standard Model (SM) is an effective field theory, valid up to a scale $\Lambda$. The excellent agreement between the theory and experiment fixes this scale on a few $\mathrm{TeV}$. Above this scale a new theory must solve the hierarchy problem. One possible solution to this problem was recently given [1] by the Little Higgs models. In these models, the Standard Model Higgs particle is viewed as a pseudo-Goldstone boson of a global symmetry group. In a first stage, this symmetry is spontaneously broken and the Higgs is a massless particle. A new collective symmetry breaking then gives a mass to the Higgs. The net result is that the new particles and couplings cancel exactly the bad behavior of the Standard Model divergent diagrams giving a light mass to the Higgs fields. Recently several proposals for a Little Higgs model have been made. Their main difference is in the choice of the new global symmetry. One explicit model, with the fewer number of new parameters as possible, was named as the Littlest Higgs model (LHM). It is constructed using an $S U(5) / S O(5)$ coset: a gauged $(S U(2) \otimes U(1))^{2}$ is broken to its subgroup $S U(2)_{L} \otimes U(1)_{Y}$. A detailed study of this model is done in reference [2].

The LHM introduces a few extra heavy particles whose typical mass scale is of order $f \equiv \Lambda / 4 \pi$, where $f$ is a decay constant of the pseudo-Goldstone boson. There are four massive extra gauge bosons and they are mixed with the SM gauge bosons after the electroweak symmetry breaking. As a result, the set of extra gauge bosons at the weak scale consists of electrically neutral states $\left(A_{H}, Z_{H}\right)$ and charged states $\left(W_{H}^{ \pm}\right)$. Among them, the $A_{H}$ boson is the lightest so that it is expected to be discovered at future collider experiments rather early. In this work, we discuss the possibility of detecting the new neutral gauge boson $A_{H}$ in the future ILC experiment with $\sqrt{s}=500 \mathrm{GeV}$ and $\mathcal{L}_{\text {int }}=340 \mathrm{fb}^{-1}$. Since there are many models with new interactions and particles it will be necessary to have very clear signatures for each model. For this, we study the forward-backward and left-right asymmetries in the processes $e^{+} e^{-} \longrightarrow f+\bar{f}$, with $f=\mu, c$ and $b$.

\section{FORWARD-BACKWARD ASYMMETRIES}

In order to compare the LHM predictions with other models we have employed the canonical $\eta, \chi, \psi$ superstring inspired $E_{6}$ models [3] and the left-right models [4]. We have performed the analytical calculation with the CompHep package [5]. For the LHM free parameters $c(\cos \theta)$ and $c^{\prime}\left(\cos \theta^{\prime}\right)$ we used the ranges of $0 \sim 0.5$ and $0.65 \sim 0.73$, respectively, preferred by the electroweak precision data. The Figures below show the forward-backward asymmetries $\left(A_{F B}\right)$ for the LHM $\left(c=0.3\right.$ and $\left.c^{\prime}=0.71\right)$, compared to other $Z^{\prime}$ models, for the processes $e^{+} e^{-} \longrightarrow \mu^{+}+\mu^{-}$, and $e^{+} e^{-} \longrightarrow b+\bar{b}$. The same comparison was done for the $e^{+} e^{-} \longrightarrow c+\bar{c}$ channel. These results are shown in Figs. 1, 2 and 3. (For more details, see [6].)

\section{LEFT-RIGHT ASYMMETRIES}

The left-right asymmetry $\left(A_{L R}\right)$ was obtained considering polarized beams. The degrees of polarization of the electron and positron beams were taken to be $90 \%$ and $60 \%$, respectively. Figures 4, 5 and 6 show the left-right asymmetries for the LHM $\left(c=0.3\right.$ and $\left.c^{\prime}=0.71\right)$, compared to other $Z^{\prime}$ models, for the processes $e^{+} e^{-} \longrightarrow \mu^{+}+\mu^{-}, e^{+}+e^{-} \longrightarrow b+\bar{b}$ and $e^{+}+e^{-} \longrightarrow c+\bar{c}$. 


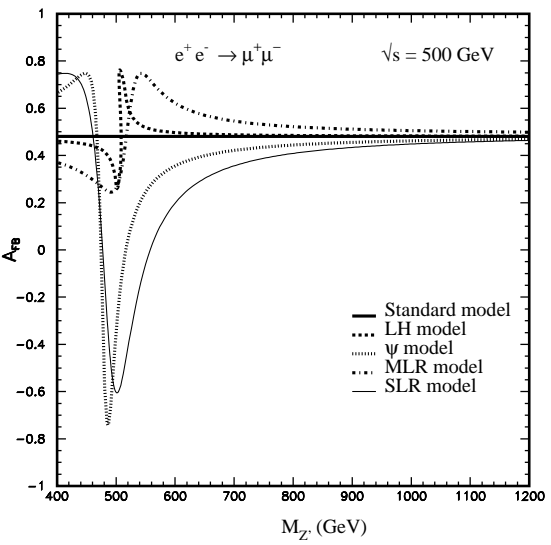

FIG. 1: Forward-backward asymmetry versus $M_{Z^{\prime}}\left(M_{A_{H}}\right)$ for $\sqrt{s}=$ $500 \mathrm{GeV}$ in $e^{+}+e^{-} \longrightarrow \mu^{+}+\mu^{-}$for some models.

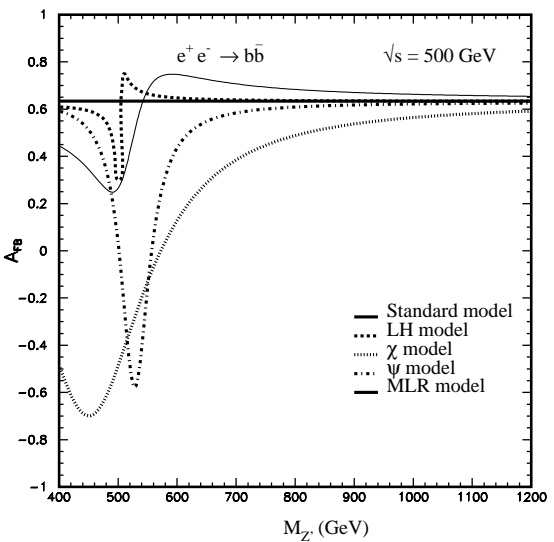

FIG. 2: Forward-backward asymmetry versus $M_{Z^{\prime}}\left(M_{A_{H}}\right)$ for $\sqrt{s}=$ $500 \mathrm{GeV}$ in $e^{+}+e^{-} \longrightarrow b+\bar{b}$ for some models.

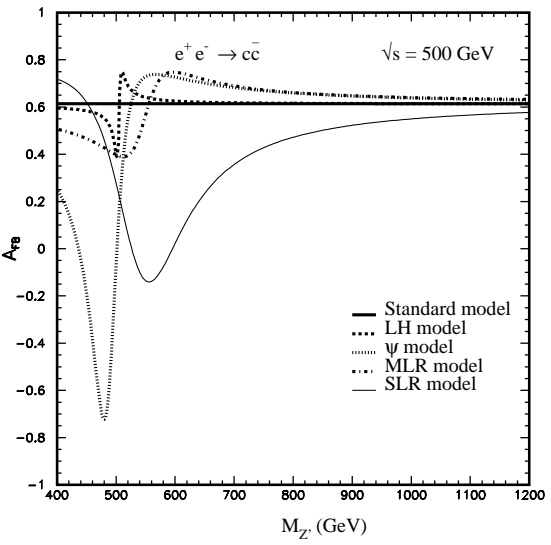

FIG. 3: Forward-backward asymmetry versus $M_{Z^{\prime}}\left(M_{A_{H}}\right)$ for $\sqrt{s}=$ $500 \mathrm{GeV}$ in $e^{+}+e^{-} \longrightarrow c+\bar{c}$ for some models.

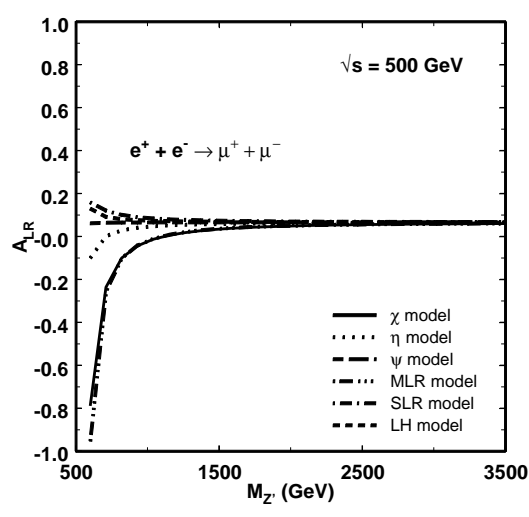

FIG. 4: Left-right asymmetry in $e^{+}+e^{-} \longrightarrow \mu^{+}+\mu^{-}$versus $M_{Z}$ $\left(M_{A_{H}}\right)$ for $\sqrt{s}=500 \mathrm{GeV}$ for some models.

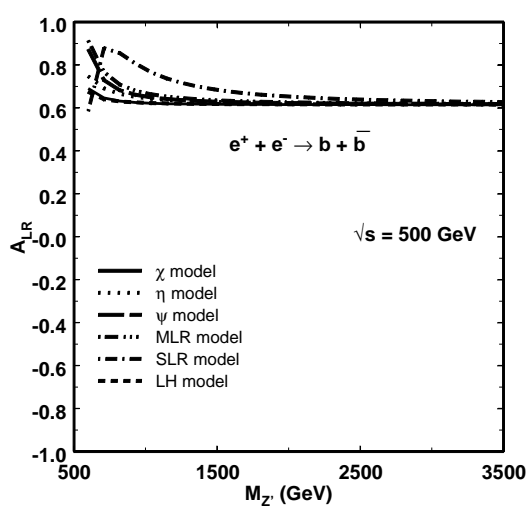

FIG. 5: Left-right asymmetry in $e^{+}+e^{-} \longrightarrow b+\bar{b}$ versus $M_{Z^{\prime}}\left(M_{A_{H}}\right)$ for $\sqrt{s}=500 \mathrm{GeV}$ for some models.

\section{LIMITS ON THE NEW GAUGE BOSON MASS}

The realistic observability upper limits with $95 \%$ C. L. of the new gauge boson $A_{H}$ on the free parameter $c^{\prime}$ was estimated by performing an $\chi^{2}$ analysis. We compared the angular distribution $d \sigma / d \cos \theta$ predicted by the LHM with the corresponding SM expectation. Supposing that the experimental data in the fermion pair production will be described by the SM predictions, we defined a one-parameter $\chi^{2}$ estimator

$$
\chi^{2}=\sum_{i=1}^{n_{b}}\left(\frac{N_{i}^{S M}-N_{i}^{L H M}}{\Delta N_{i}^{S M}}\right)^{2},
$$

where $N_{i}^{S M}$ is the number of SM events collected in the $i^{t h}$ bin, $N_{i}^{L H M}$ is the number of events in the $i^{t h}$ bin as predicted by the LHM, and $\Delta N_{i}^{S M}=\sqrt{\left(\sqrt{N_{i}^{S M}}\right)^{2}+\left(N_{i}^{S M} \varepsilon\right)^{2}}$ the correspond- 


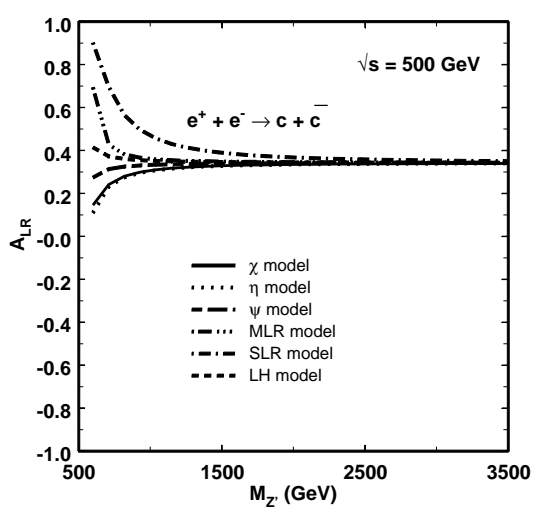

FIG. 6: Left-right asymmetry in $e^{+}+e^{-} \longrightarrow c+\bar{c}$ versus $M_{Z^{\prime}}\left(M_{A_{H}}\right)$ for $\sqrt{s}=500 \mathrm{GeV}$ for some models.

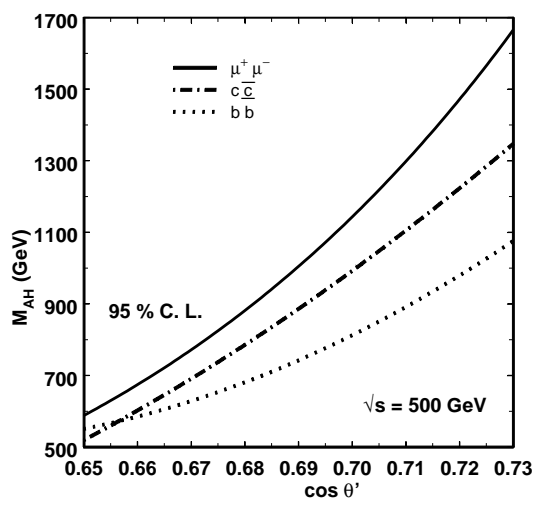

FIG. 7: $M_{A_{H}}$ upper bounds $\left(95 \%\right.$ C.L.) as a function of $\cos \theta^{\prime}$ in $e^{+}+e^{-} \longrightarrow f+\bar{f}$, where $f=\mu, c$ and $b$ for $\sqrt{s}=500 \mathrm{GeV}$ for LHM.

ing total error, which combines in quadrature the Poissondistributed statistical error with the systematic error. We took $\varepsilon=5 \%$ as the systematic error in our calculation. We consid- ered the muon, charm and bottom detection efficiency as $95 \%$, $60 \%$ and $35 \%$ respectively.

For leptons $\mu^{+} \mu^{-}$and quarks $\bar{c} c, \bar{b} b$ in final states, $\sqrt{s}=500$ $\mathrm{GeV}$ and $\sqrt{s}=1 \mathrm{TeV}$, our results are displayed in Figures 7 and 8 , respectively.

\section{v. CONCLUSIONS}

The asymmetries showed that they can be used to distinguish the LHM from the others $Z^{\prime}$ models, with some restrictions, since the curves intercept each other for different $A_{H}$ mass values below $700 \mathrm{GeV}$. For masses above $700 \mathrm{GeV}$ the

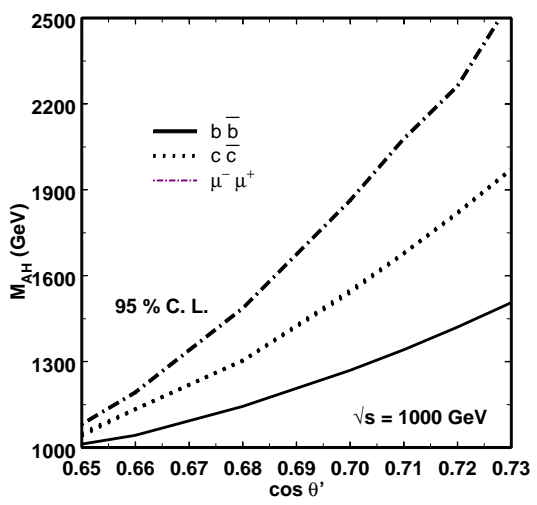

FIG. 8: $M_{A_{H}}$ upper bounds (95\% C.L.) as a function of $\cos \theta^{\prime}$ in $e^{+}+e^{-} \longrightarrow f+\bar{f}$, where $f=\mu, c$ and $b$ for $\sqrt{s}=1 \mathrm{TeV}$ for LHM.

asymmetries curves approach the SM prediction while for polarized beams this occurs at $900 \mathrm{GeV}$. Using the angular distributions it is possible to obtain more restrictive mass limits than using the total cross-sections as done by [7]. Each model generates an angular distribution shape even when the number of events are not so different. In this way there are contributions for the mass limits and model comparisons not only from the total of events but also from their specific angular distributions.

Acknowledgments: CNPq, FAPERJ and FAPEMIG.
[1] N. Arkani-Hamed, A. G. Cohen, and H. Georgi, Phys. Lett. B 513, 232 (2001) [arXiv:hep-ph/0105239].

[2] T. Han, H. E. Logan, B. McElrath, and L. T. Wang, Phys. Rev. D 67, 095004 (2003) [ar-Xiv:hep-ph/0301040].

[3] Particle Data Group, Phys. Lett. B 592, 1 (2004).

[4] F. M. L. Almeida Jr., Y. A. Coutinho, J. A. Martins Simões, J. Ponciano, A. J. Ramalho, S. Wulck, and M. A. B. do Vale, Eur. Phys. J. C 38, 115 (2004).

[5] A. Pukhov, E. Boos, M. Dubinin, V. Edneral, V. Ilyin, D. Kovalenko, A. Kryukov, V. Savrin, S. Shichanin, and A. Semenov,
"CompHEP"- a package for evaluation of Feynman diagrams and integration over multi-particle phase space. Preprint INP MSU 98-41/542 [arXiv:hep-ph/9908288]

[6] F. M. L. Almeida Jr., Y. A. Coutinho, J. A. Martins Simões, A. J. Ramalho, S. Wulck, and M. A. B. do Vale, ar-Xiv:hep$\mathrm{ph} / 0601102$.

[7] Chong-Xing Yue, Wei Wang, and Feng Zhang, Nucl. Phys. B 716, 199 (2005). 